\title{
Residual Magnetic Anomalies Mapping from CHAMP Observations
}

\author{
M. C. Berguig1, S. Bouraoui' ${ }^{1}$, Y. Cohen ${ }^{2}$ \\ ${ }^{1}$ Département de Géophysique FSTGAT, University of Science and Technology Haouari Boumedienne, Algiers, \\ Algeria \\ ${ }^{2}$ Institut de Physique du Globe de Paris, Paris, France \\ Email: berguig@ipgp.fr, seyfbouraoui@gmail.com,cohen@ipgp.fr
}

Received 16 February 2016; accepted 15 May 2016; published 18 May 2016

Copyright (C) 2016 by authors and Scientific Research Publishing Inc.

This work is licensed under the Creative Commons Attribution International License (CC BY).

http://creativecommons.org/licenses/by/4.0/

(c) (†) Open Access

\begin{abstract}
CHAMP high-quality vector magnetometer observations collected from July 2000 to September 2010 have been used to map the residual vector magnetic anomaly fields. This field is so called the lithospheric magnetic field which is the result of two contributions of the induced and the remanent magnetization. It is therefore essential to study the magnetic properties of the crustal rocks. Isolating this field from the other contributions, interpreting and even defining are however difficult and still debated. We investigate how to identify and separate the lithospheric vector magnetic field $\Delta X, \Delta Y$ and $\Delta Z$ from other contributions. For this purpose we use selected night magnetic data from which we remove a model field of degree 16 and external model field of degree 2 developed by spherical harmonics analysis. Concerning the induced lithospheric field which is assumed to be aligned with the internal dipole was also removed. To minimize the secular variation effects, we calculated internal models for each two months. The method developed here has been successfully applied to isolate lithospheric field produced by remanent magnetizations from CHAMP satellite data. The resolution and altitude measurements make it very hard to map short wavelength crustal magnetic anomalies. The large-scale strong magnetic anomalies detected using this technique are in agreement with previous global magnetic maps. These anomalies appear with an amplitude of about $10 \mathrm{nT}$ at satellite altitude such as Bangui's anomaly.
\end{abstract}

\section{Keywords}

Magnetic Field, Lithospheric Anomalies, External Field, CHAMP Measurements, Remanent Magnetization 


\section{Introduction}

The Earth's observed magnetic field at surface or in orbital altitude can be separated into two contributions: external and internal contributions varying in spatial and temporal scales [1]. The external contribution part is produced by a complex system of currents circulating in the ionosphere and magnetosphere and their interactions with solar wind. The internal field is the superposition of two fields: a main field generated by the currents in the liquid outer core and a lithospheric field generated by induced currents and remanent magnetization of crust's rocks where temperatures are well below the Curie isotherm. Most of the remanent magnetic contributions cancel out at the spacecraft altitude and the induced part dominates above continental domains [2] [3].

In spite of the large sets of magnetometer data from ground to satellites altitudes, it remains very controversy to identify separately each part of source contributions from the observed magnetic field. The external magnetic field is relatively a small portion of the observed field which represents only $1 \%$ of the total field but this parasite component must be removed to determine the exact properties of the internal field [4]. Most algorithms and methods have been developed to extract and verify the crustal magnetic anomalies especially from satellite magnetometer measurements [2] [3] [5]-[7]. The analysis of the spatial distribution of a lithospheric magnetic field shows that the anomalies significantly decrease at the satellites altitudes: the small-scale anomalies are smoothed, and only the large-scale and strong anomalies with wavelength of $400-500 \mathrm{~km}$ and intensity of a few dozen $\mathrm{nT}$ were detected. The satellite measurements are poorly sensitive to small-scale structures, making the satellite data favorable for identification of the regional lithospheric anomalies [2]. The traditional techniques used nowadays to map lithospheric magnetic anomalies were based essentially on models developed using Gauss harmonic coefficients (see for example [2] [3] [7]-[9]).

In this paper we present new methodology to extract the magnetic contributions of the lithosphere from a set of satellite magnetometer vector data. To do so, we adapted new techniques to map the vector magnetic field of the lithosphere using magnetic data collected by CHAMP satellite for more than ten years. First, magnetic data were separated into daily and nighttime data using local time transformations and then less disturbed data by external fields were selected using geomagnetic indices and their derivatives. Monthly selected night data were inverted to determine a core field of 16 degrees; this internal field was removed from previously selected data to obtain residual fields. In order to improve our results, these data representing the residual were denoised from external persistent fields using inversion methods based on harmonic analysis developed by [5] [10] similar to that described by [11] and [12]. After all the data obtained were used to determine residual vector magnetic anomalies $\Delta X, \Delta Y$ and $\Delta Z$.

In the next section we describe the data selection and processing algorithms used to remove the internal magnetic data from secular variations. In Section 3 we present our methodology to map magnetic anomalies of the earth's lithosphere at satellite altitudes. In Section 4 we discuss some results arising from our methods used in this paper. Section 5 then concludes with some observations on results and future for this work.

\section{Data Selection and Processing}

The magnetic measurements on board CHAMP satellite operated continuously from July 2000 to September 2010 were used to study lithospheric magnetic anomalies. This satellite have been operating in nearly polar circular orbits of 93.55 minutes with an inclination of $87.17^{\circ}$ [13]. The measurement accuracy for the scalar magnetic data was $\pm 0.5 \mathrm{nT}$, and that for the vector was $\pm 3 \mathrm{nT}$ [13]. The altitudes have been decreasing between 350 and $450 \mathrm{~km}$ at all local times and the data are acquired every second which corresponds to the $7 \mathrm{~km}$ of spatial longitudinal resolution [2] [14]. These orbital parameters allow a high resolution and improve the mapping of the earth magnetic fields [3] [7] [15]. In this paper, both vector magnetic and scalar data of level 3 were processed to map the geomagnetic fields internal variations. The crucial processing step in this kind of work is the selection of less disturbed data. Thereby the main objective of the proposed selection data methods is to obtain in first time a set of measurements related to their sources. In our case, we attempted to separate the effects of external fields from the internal field components using global geomagnetic indices such as $K p$ and Dst. The algorithm used here for selecting quiet magnetic measurements works respecting the following steps:

- Reduce the effect of the external magnetic field using data collected in the night side at low altitudes for local time LT between $22 \mathrm{~h} 00$ and $06 \mathrm{~h} 00$.

- These selected data were compared to geomagnetic indices $K p$ and $D s t$ and data corresponding to $K p \leq 1+$ 
and $|D s t|<=15 \mathrm{nT}$ with $\frac{\partial D s t(T)}{\partial t} \leq 3 \mathrm{nT} \cdot \mathrm{hr}^{-1}$ were selected.

- The vector data measured below $\pm 55^{\circ}$ geomagnetic latitude were considered in order to remove auroral effects where the scalar data above this latitude were used.

The all CHAMP vector and scalar data obtained using these selection criteria were split into monthly files to be used for modeling internal field. Several tests were executed in order to find the most optimal coverage corresponding to the shortest possible duration. More the period is shorter, the effects of the external field, the induced magnetization and secular variations were reduced. So, the most appropriate shortest period used to elaborate consistent spherical harmonics models is corresponding to sets of data covering at least a large band of magnetic equator in order to reduce Backus effect [16]. The data computed with our inversion program has given global two-months models for internal field which are in good agreement with accepted international models such as IGRF-11. Our proposed algorithm minimizes in the fact the secular variation which is visible for periods more than six months [1] [17]. The Figure 1 shows vector geomagnetic components before and after applying selection criteria for the days of 01-05-2007.

The Figure 2 shows an example of the data coverage used by inversion in order to determine the main field of degree 16. The data presented in this figure were corresponding to august and September of year 2009.

\section{Modeling Methodology}

The spherical harmonic analysis is one of the best way to describe the geomagnetic field related to its external and internal sources [18] [19]. The Earth's magnetic field is derived from the magnetic potential $V$ verifying Laplace's equation and is expressed in nanotesla (nT). In the geocentric reference the geomagnetic field can be expressed using scalar potential by:

$$
\boldsymbol{B}^{\text {mod }}=-\nabla V(r, \theta, \varphi, t)
$$
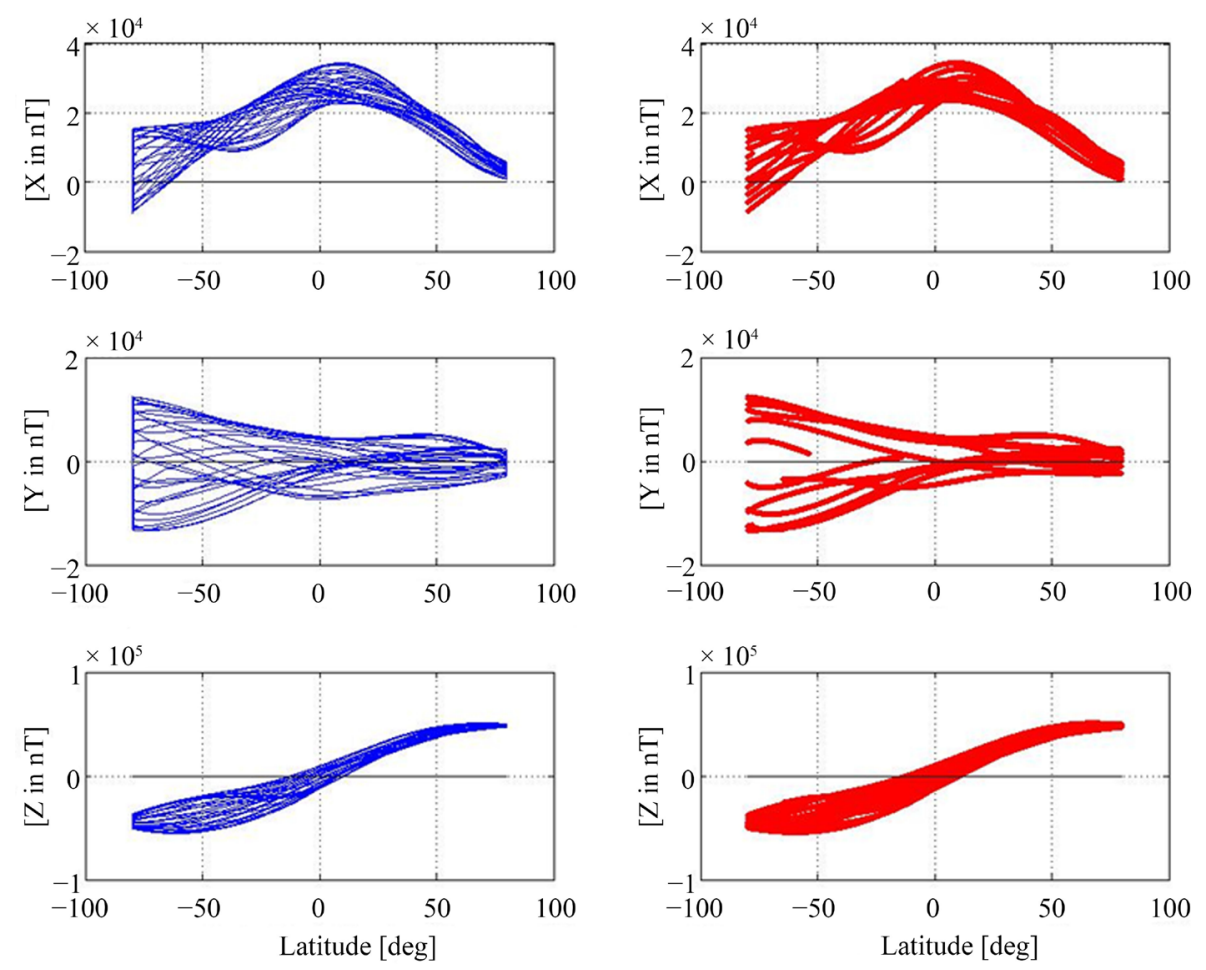

Figure 1. Example of applying selection criteria for the day 01-05-2007. Left: Variation of raw vector geomagnetic components (before selecting). Right: Selected data for $K p=1+$ and $|D s t| \leq 20 \mathrm{nT}$. 


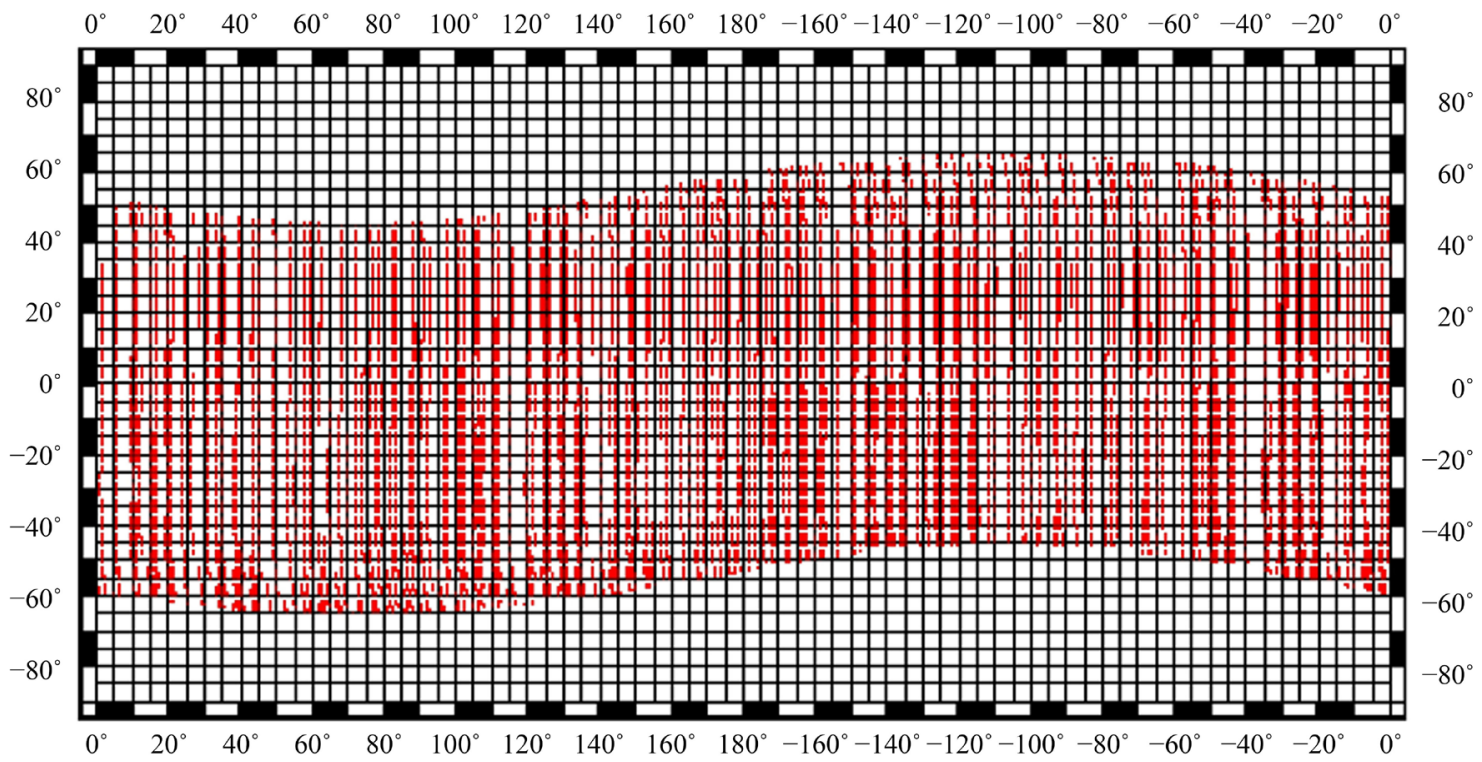

Figure 2. Example of the coverage of selected data for the two months 08 and 09 of 2009 using in inversion. The projection system is the Orthographic centered on longitude $180^{\circ}$.

It is well that the potential $V$ can be written in term of spherical harmonic expansion as follows (in [19]):

$$
V(r, \theta, \varphi, t)=V_{\text {int }}(r, \theta, \varphi, t)+V_{\text {ext }}(r, \theta, \varphi, t)
$$

The $V_{i n t}$ is the internal potential and $V_{e x t}$ potential which are expressed by these relations:

$$
\begin{aligned}
& V_{\text {int }}(r, \theta, \varphi, t)=R_{E} \sum_{n_{i}=1}^{N_{i} \max } \sum_{m=0}^{n_{i}}\left(\frac{R_{E}}{r}\right)^{n+1}\left[g_{m}^{n}(t) \cos (m \varphi)+h_{m}^{n}(t) \sin (m \varphi)\right] P_{m}^{n}(\theta) \\
& V_{\text {ext }}(r, \theta, \varphi, t)=R_{E} \sum_{n_{i}=1}^{N_{i} \max } \sum_{m=0}^{n_{i}}\left(\frac{r}{R_{E}}\right)^{n}\left[q_{m}^{n}(t) \cos (m \varphi)+s_{m}^{n}(t) \sin (m \varphi)\right] P_{m}^{n}(\theta)
\end{aligned}
$$

where $g_{m}^{n}, h_{m}^{n}$ and $q_{m}^{n}, s_{m}^{n}$ are respectively internal and external Gauss coefficients expressed in $\mathrm{nT}, R_{E}$ is spherical earth of radius equal to $6371.2 \mathrm{~km}$. The main objective of modeling is to minimize this quantity:

$$
\sigma^{2}=\sum_{i=1}^{\text {Nobs }} W_{i}\left(\boldsymbol{B}_{i}^{\text {obs }}\left(r_{i}, \theta_{i}, \varphi_{i}, t_{i}\right)-\boldsymbol{B}_{i}^{\text {mod }}\left(r_{i}, \theta_{i}, \varphi_{i}, t_{i}\right)\right)^{2}
$$

$\boldsymbol{B}^{\text {obs }}$ is the observed field, $W$ is a function of weights which depends in $\sin (\theta)$. We have developed an algorithm mixing c and matlab which can invert simultaneously both scalar $(F)$ and vector magnetic data $(X, Y$ and $Z$ ). Thus, for our purpose the all CHAMP vector and scalar data selected were split into two monthly files to be used for modeling geomagnetic core field. Global models of this field for 16 degrees of spherical harmonics expansion were established for each two months for all period of CHAMP mission. Thus, the residual fields were calculated by removing the core, magnetospheric and induced fields from previously selected night data.

This approach of modeling each two month is used to minimize the effects of the secular variation which appear in data for periods more than 6 months [1] [17]. The Figure 3 shows the power spectrum of the core field for corresponding to the year 2009 for each two-months.

From Figures 4-6 are represented the three components of the core field obtained by inversion. The map of the $Z$ component show the dominant dipole configuration of internal geomagnetic field.

The Figures 7-9 show the result of application of our algorithms to the selected data.

\section{Results and Discussion}

The method presented here to map lithosphere magnetic field is a useful alternative to more conventional 


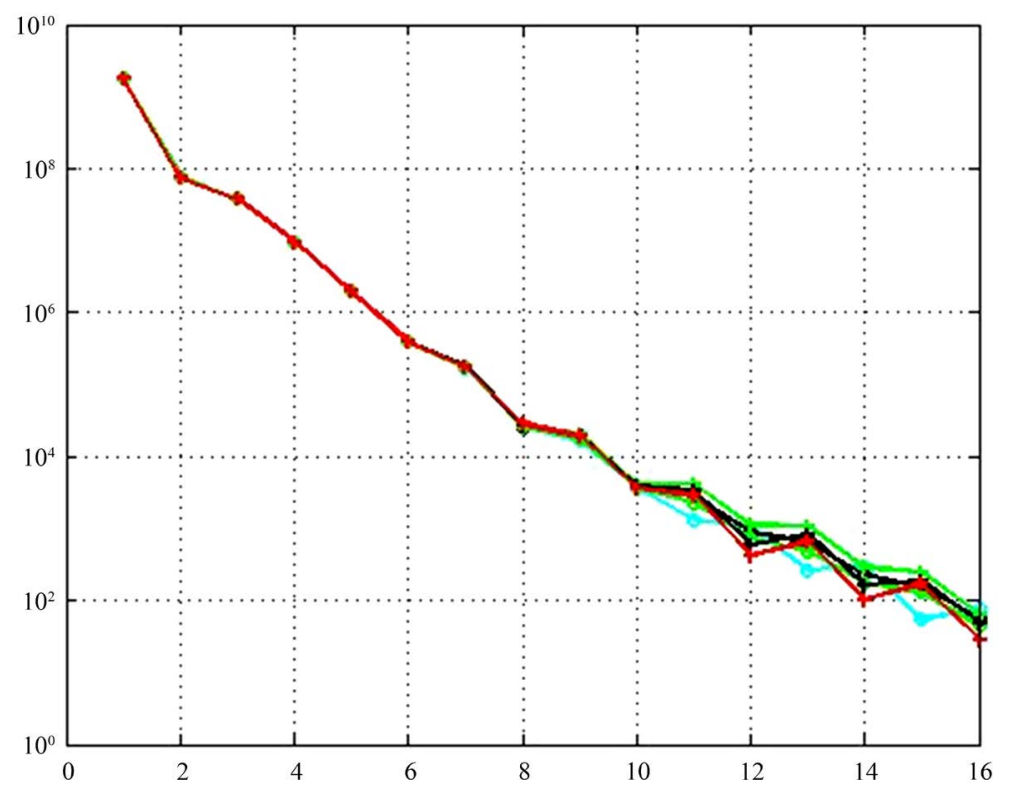

Figure 3. The power spectrum of core field obtained by inversion for the year 2009 for each two-months.

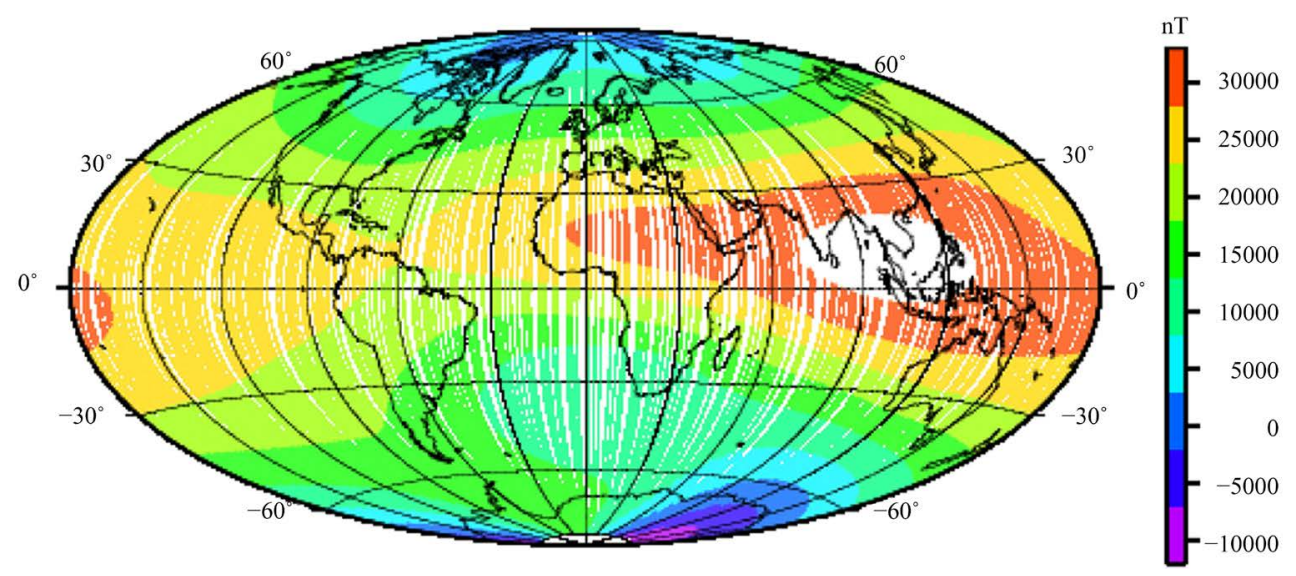

Figure 4. Maps showing the modeled component $X$ at satellite altitude for the January 2009. The system of projection is the Hammer-Aitoff centered on $0^{\circ}$. The color scale is nT.

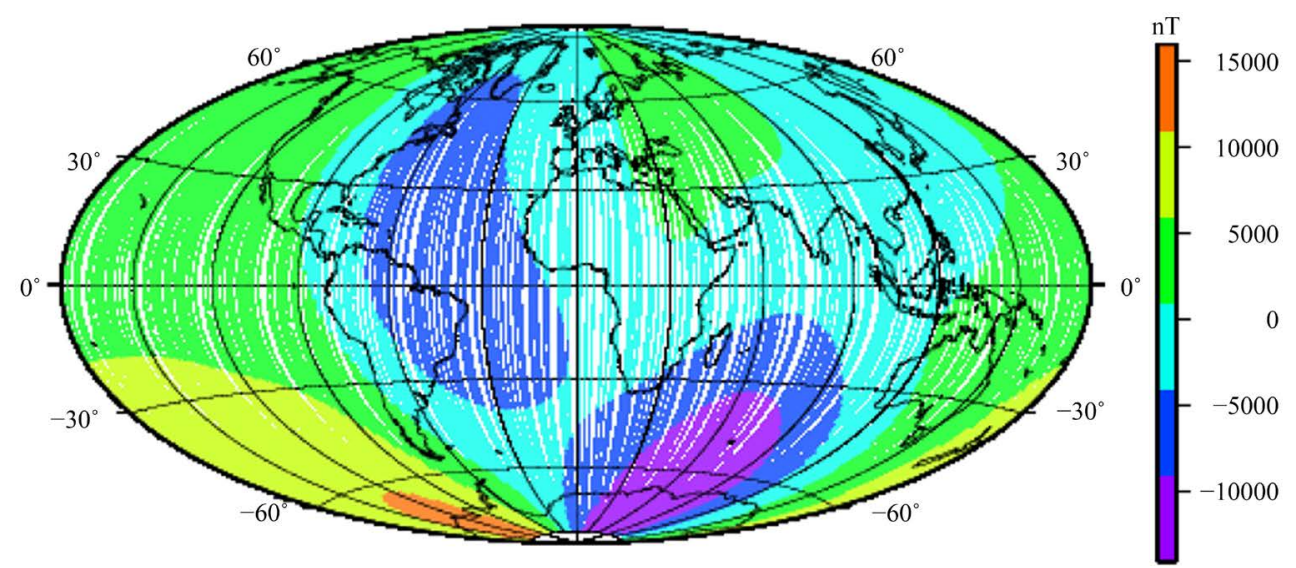

Figure 5. Maps showing the modeled component $Y$ at satellite altitude for the January 2009. The system of projection is the Hammer-Aitoff centered on $0^{\circ}$. The color scale is nT. 


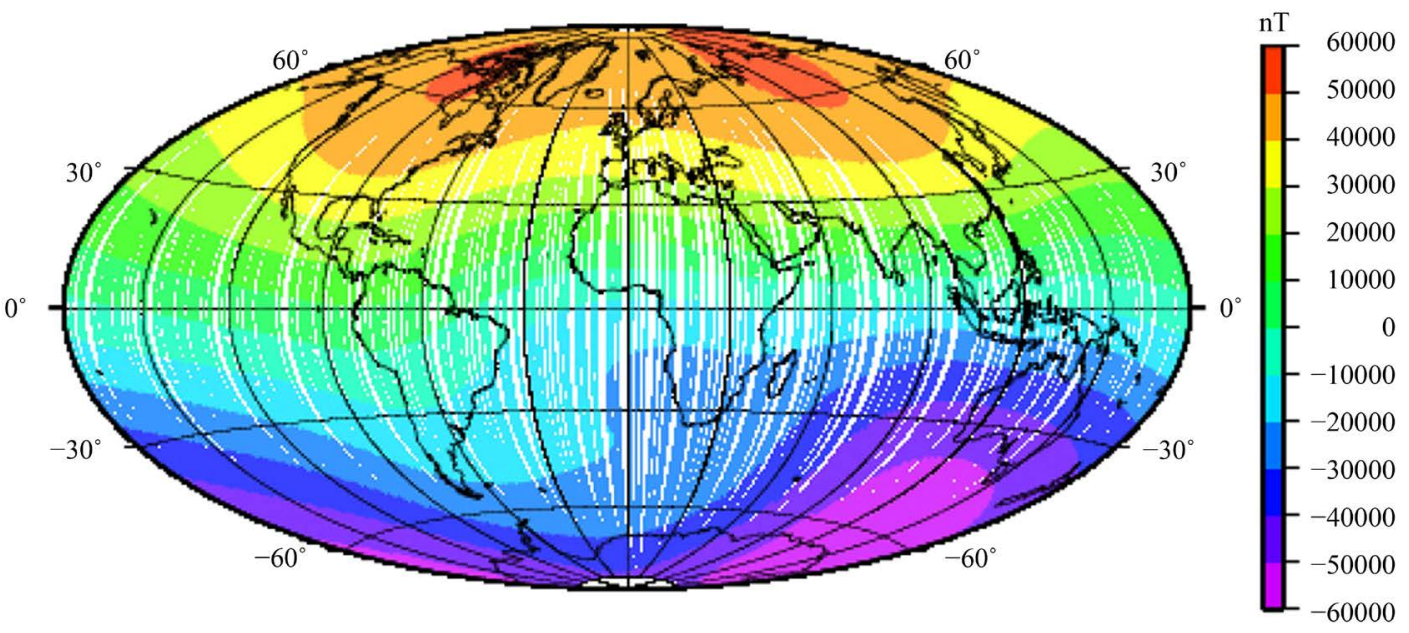

Figure 6. Maps showing the residual component $Z$ at satellite altitude for the year 2009. The system of projection is the Hammer-Aitoff centered on $0^{\circ}$. The color scale is nT.

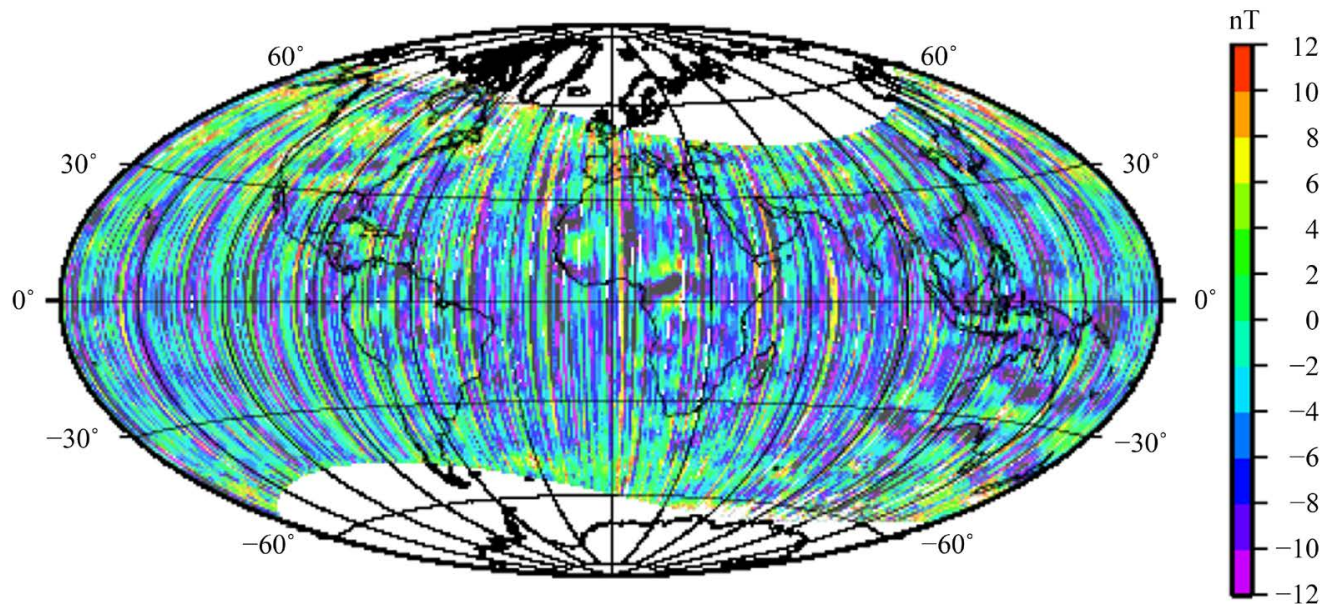

Figure 7. Maps showing the residual component $X$ at satellite altitude below $\pm 55^{\circ}$ of geomagnetic latitude for the selected year 2009. The system of projection is the Hammer-Aitoff centered on $0^{\circ}$. The color scale is nT.

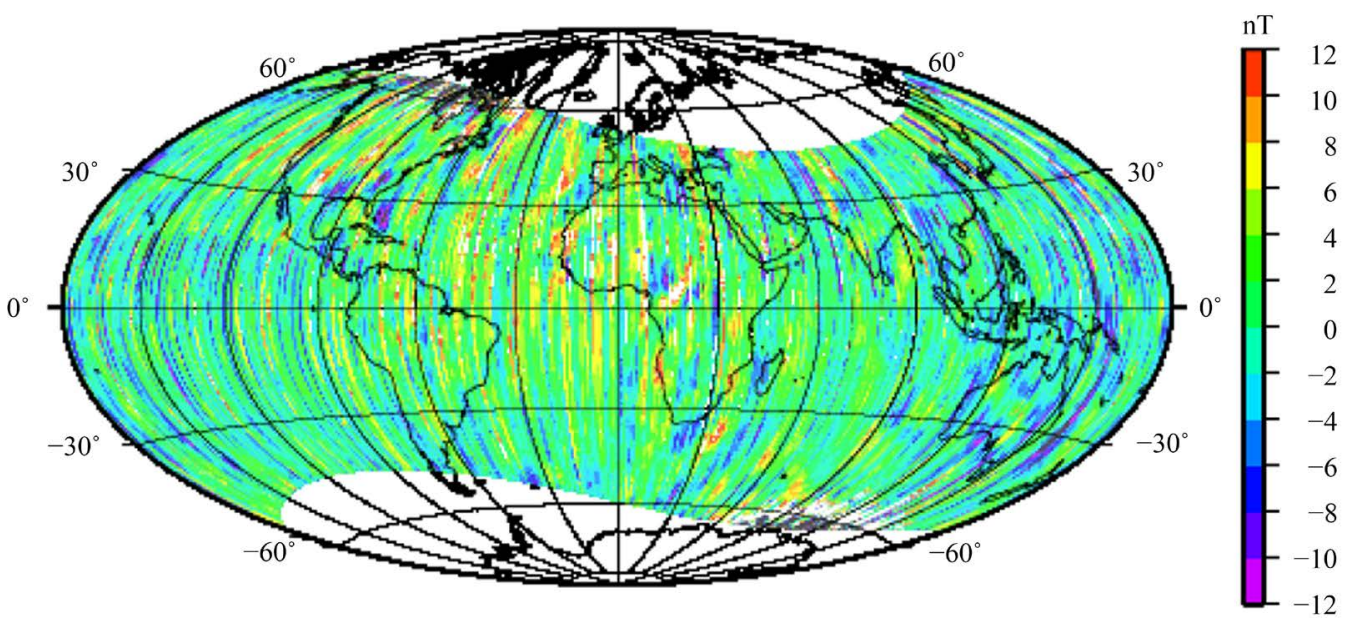

Figure 8. Maps showing the residual component $Y$ at satellite altitude below $\pm 55^{\circ}$ of geomagnetic latitude for the selected year 2009. The system of projection is the Hammer-Aitoff centered on $0^{\circ}$. The color scale is $\mathrm{nT}$. 


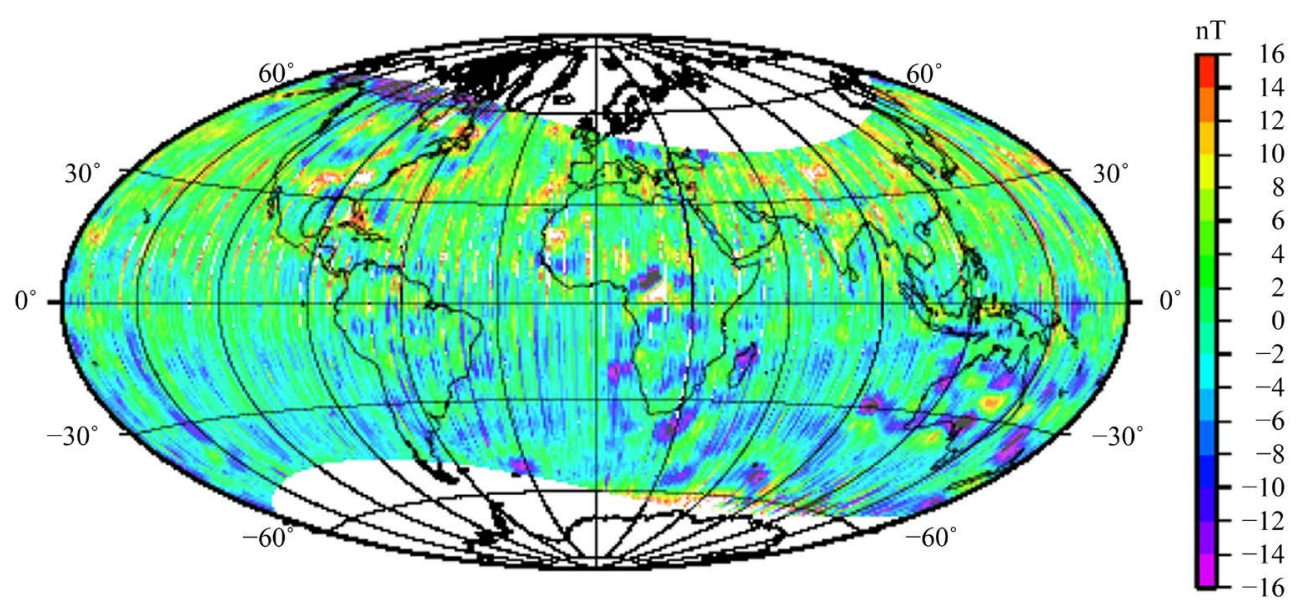

Figure 9. Maps showing the residual component $Z$ at satellite altitude below $\pm 55^{\circ} \operatorname{irc}$ of geomagnetic latitude for selected the year 2009. The system of projection is the Hammer-Aitoff centered on $0^{\circ}$. The color scale is nT.

spherical harmonic models especially for large-scale anomalies. New maps of crustal magnetic anomalies field at variable satellite altitudes have been successfully constructed by inverting CHAMP data, based on: - Using a night-side selection of less disturbed magnetic observations from 2000 to 2010 we produced models of the geomagnetic field in which the internal fields of 16 degrees are modeled for periods of two-month using harmonics analysis. The amplitude and the direction of the magnetic field obtained by this method are in good agreement with accepted models such as IGRF models.

The residual fields obtained directly from CHAMP observation represent large-scale lithosphere magnetic anomalies.

The interpretation of this residual magnetic field is still difficult because there is non-uniqueness physical description of the geomagnetic crust.

- In spite of the data selection criteria, the effect of the external field remains important in longitudinal direction.

- Only strong anomalies such a Bangui anomaly are clear in this residual distribution.

- The anomaly associated to the West African craton, though identifiable, is also strongly affected by noise.

- Altitude and spatial resolution make mapping of crustal field more difficult, which requires integration of other complementary data such as land and aeromagnetic measurements.

- The satellite observations provide an excellent global coverage that allows use to obtain monthly models for core field and its secular variation.

Our methods are not able to detect small anomalies, and new models for large degrees will be developed in further work. In the meantime, significant efforts are still being made to improve our maps and to better characterize these anomalies with the recent SWARM observations on the one hand, and near-surface data on the other hand. The use of parallel algorithms to extend the degree of harmonic expansion allows us a best modeling of the magnetic lithosphere for small-scale anomalies.

The Earth's magnetized lithosphere and the complexity of its magnetic sources and their interactions with external fields, and the variety of measurements need the construction of models for highly degrees in order to understand the extent to which the residual field may be interpreted. In addition, to best understand and interpret the residual magnetic field maps it was also the purpose of our work to join the other maps using calculated models from the different procedures and combining them into the unifying procedure to present a consistent mapping of lithospheric magnetic field.

\section{References}

[1] Langel, R.A. (1993) The Use of Low Altitude Satellite Data-Bases for Modeling of Core and Crustal Fields and the Separation of External and Internal Fields. Surveys in Geophysics, 14, 31-87. http://dx.doi.org/10.1007/BF01044077

[2] Maus, S., Rother, M., Holme, R., Lühr, H., Olsen, N. and Haak, V. (2002) First Scalar Magnetic Anomaly Map from 
CHAMP Satellite Data Indicates Weak Lithospheric Field. Geophysical Research Letters, 29. http://dx.doi.org/10.1029/2001gl013685

[3] Thébault, E., Purucker, M., Whaler, K.A., Langlais, B. and Sabaka, T.J. (2010) The Magnetic Field of the Earth’s Lithosphere. Space Science Reviews, 55, 95-127. http://dx.doi.org/10.1007/s11214-010-9667-6

[4] Olsen, N., Glassumier, K.H. and Jia, X. (2010) Separation of the Magnetic Field into External and Internal Parts. Space Science Reviews, 152, 135-157. http://dx.doi.org/10.1007/s11214-009-9563-0

[5] Berguig, M.C., Hamoudi, M., Cohen, Y. and Le Mouël, J.L. (2011) Validate Global Mapping of Internal Lunar Magnetic Field. Arabian Journal of Geosciences, 6, 1063-1072. http://dx.doi.org/10.1007/s12517-011-0406-4

[6] Mandea, M. and Langlais, B. (2002) Observatory Crustal Magnetic Biases during MAGSAT and Oersted Satellite Missions. Geophysical Research Letters, 29.

[7] Olsen, N., Lühr, H., Finlay, C.C., Sabaka, T.J., Michaelis, I., Rauberg, J. and Tøffner-Clausen, L. (2014) The CHAOS4 Geomagnetic Field Model. Geophysical Journal International, 197, 815-827. http://dx.doi.org/10.1093/gji/ggu033

[8] Maus, S., Lühr, H., Balasis, G., Rother, M. and Mandea, M. (2005) Introducing POMME, the Potsdam Magnetic Model of the Earth. In: Reigber, C., Li'lhr, H., Schwintzer, P. and Wickert, J., Eds., Earth Observation with CHAMP, Results from Three Years in Space, Springer, Berlin-Heidelberg, 293-298. http://dx.doi.org/10.1007/3-540-26800-6_46

[9] Stockmann, R., Christopher, C.F. and Jackson, A. (2009) Imaging Earth’s Crustal Magnetic Field with Satellite Data: A Regularized Spherical Triangle Tessellation Approach. Geophysical Journal International, 179, 929-944. http://dx.doi.org/10.1111/j.1365-246X.2009.04345.x

[10] Berguig, M.C., Hamoudi, M., Cohen, Y. and Thébault, E. (2008) Lunar Magnetic Pole Positions Deduced from High Albedo Magnetic Anomalies. Asian Journal of Science Earth, 2.

[11] Lesur, V., Rother, M., Vervelidou, F., Hamoudi, M. and Thébault, E. (2013) Post-Processing Scheme for Modelling the Lithospheric Magnetic Field. Solid Earth, 4, 105-118. http://dx.doi.org/10.5194/se-4-105-2013

[12] Olsen, N., et al. (2015) The Swarm Initial Field Model for the 2014 Geomagnetic Field. Geophysical Research Letters, 42, 1092-1098. http://dx.doi.org/10.1002/2014GL062659

[13] Reigber, C., Lühr, H. and Schwintzer, P. (2002) CHAMP Mission Status. Advances in Space Research, 30, $129-134$. http://dx.doi.org/10.1016/S0273-1177(02)00276-4

[14] LeMouël J.L., Shebalin, P. and Chulliat, A. (2006) The Field of the Equatorial Electrojet from CHAMP Data. Annals of Geophysics, 24, 515-527. http://dx.doi.org/10.5194/angeo-24-515-2006

[15] Maus, S. (2010) An Ellipsoidal Harmonic Representation of Earth’s Lithospheric Magnetic Field to Degree and Order 720. Geochemistry, Geophysics, Geosystems, 11, Q06015. http://dx.doi.org/10.1029/2010gc003026

[16] Ultré-Guérard, P., Hamoudi, M. and Hulot, G. (1998) Reducing the Backus Effect Given Some Knowledge of the Dip-Equator. Geophysical Research Letters, 25, 3201-3204. http://dx.doi.org/10.1029/98GL02211

[17] Mandea, M. and Olsen, N. (2006) A New Approach to Directly Determine the Secular Variation from Magnetic Satellite Observations. Geophysical Research Letters, 33, L15306. http://dx.doi.org/10.1029/2006gl026616

[18] Blakely, R.J. (1996) Potential Theory in Gravity and Magnetic. Cambridge University Press, Cambridge.

[19] Langel, R.A. and Hinze, W.J. (1995) The Magnetic Field of the Earth's Lithosphere in Geomagnetism. Cambridge University Press, Cambridge. 